\title{
Design of a Real-Time Test Bench for UAV Servo Actuators
}

\author{
Lysandros Anastasopoulos ${ }^{1}$ and Mirko Hornung ${ }^{2}$ \\ Technical University of Munich, Faculty of Mechanical Engineering \\ Garching, 85748, Germany
}

\begin{abstract}
Reliable flight operations of Unmanned Aerial Vehicle (UAV) in the class above $25 \mathrm{~kg}$ impose strict requirements on the flight control and propulsion system. Research demonstrators however, evolve around unique aircraft configurations, while fulfilling unconventional missions. These properties increase the complexity of the on-board electromechanical devices. The latter usually rely upon Commercial off-the-shelf (COTS) components, instead of sufficiently documented, verified products. Insight into individual performance and reliability figures of these components must therefore be obtained by conducting ground-based laboratory tests. The presented test bench resembles a dynamometer for emulating aerodynamic loads on UAV servo actuators under pre-defined conditions, with the capability of monitoring and recording the relevant test parameters.
\end{abstract}

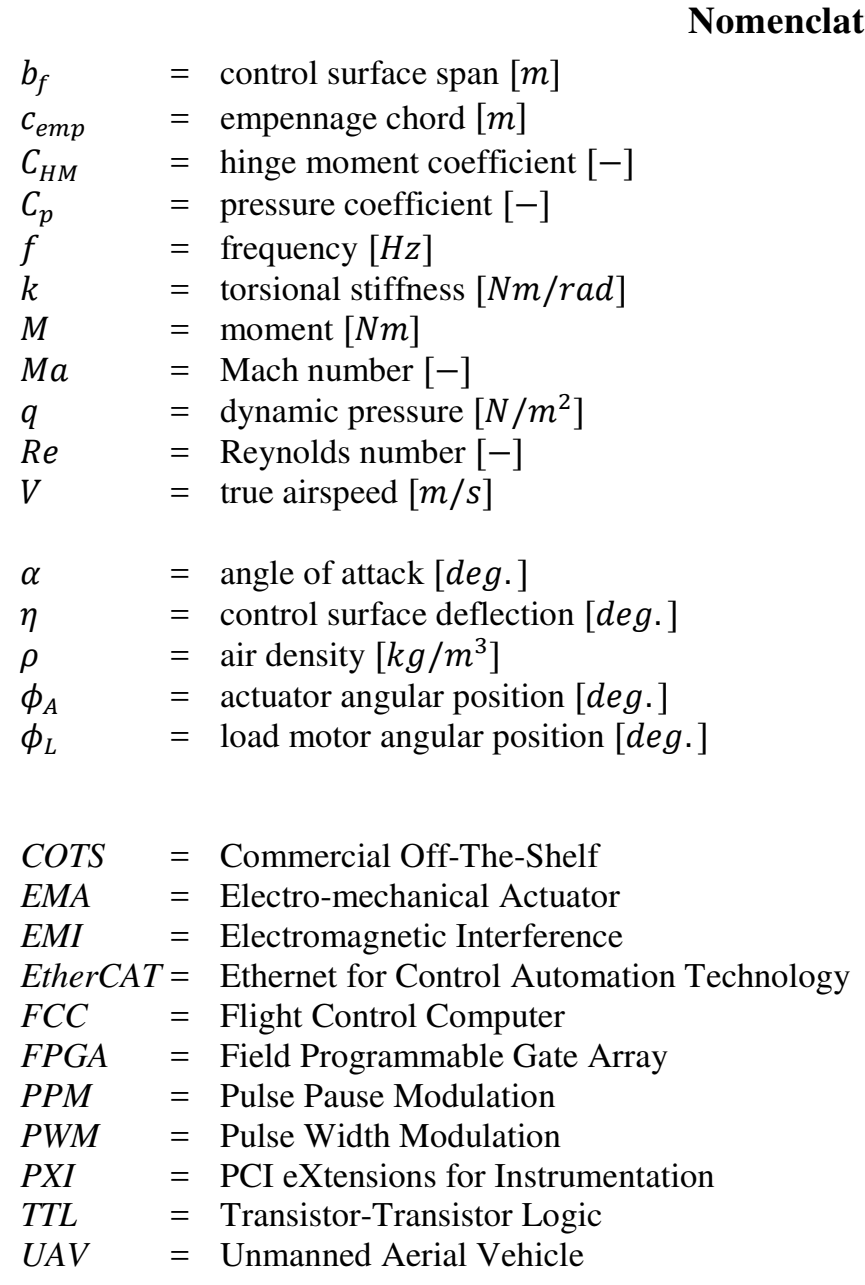

\footnotetext{
${ }^{1}$ Research associate and Ph.D. candidate, Institute of Aircraft Design. lysandros.anastasopoulos@tum.de

${ }^{2}$ Professor, Head of Institute of Aircraft Design. mirko.hornung@tum.de
} 


\title{
I. Problem statement and previous work
}

\begin{abstract}
A CTUATORS in Unmanned Aerial Vehicles are subject to significant static and dynamic loads of mechanical and thermal nature, depending on the assigned function, e.g. control surface, air brake, or landing gear movement. In order to verify the adequacy of an actuator for a specific task, a test environment is required, permitting adjustment of the position setpoint value, normally commanded by the on-board Flight Control Computer (FCC). Simultaneously, a loading device has to reproduce aerodynamic and mechanical forces expected at a given flight state. The main actuator characteristics to be quantified in this way are position-following error and achievable velocities under load. Further values of interest are peak electric current demand and average power consumption. Test results can be compared to the UAV flight control system requirements and can facilitate an initial tuning of the autopilot settings.

Traditional means of loading aircraft actuators involve gravity or spring based mechanisms. However, simultaneous adjustment of both amplitude and mean value of the load level is problematic ${ }^{1}$. A servo actuation test bench is presented in the work of Lauffs ${ }^{2}$, with particular attention to actuator redundancy and flight control architecture. In a publication of $\mathrm{Braun}^{3}$, a similar setup is described, however with a load application device identical to the aircraft actuator. A sophisticated approach for hydraulic actuators of helicopters can be found in the study of Bertucci ${ }^{4}$. The present work focuses on reproducing various rudder deflection scenarios and monitoring parameters such as applied mechanical torque and electric current. For this purpose, a loading device that possesses dynamic properties exceeding those of the actuator-under-test is chosen.
\end{abstract}

\section{Approach methodology and implementation}

Figure 1 presents the step-by-step methodology for testing Electro-mechanical Actuators (EMA), with the scope of this study highlighted in blue color. After the conceptual aircraft design phase, calculation results during preliminary design set the main flight control system requirements. Here, actuator characteristics are experimentally extracted at synthetic operating points, which can be static or transient (component testing). As special requirements and geometric factors become available in the detailed design phase, the actuator can be tested along a flight mission, in conjunction with the original FCC and on-board power supply (system testing). This aims to improve confidence in the overall system performance.

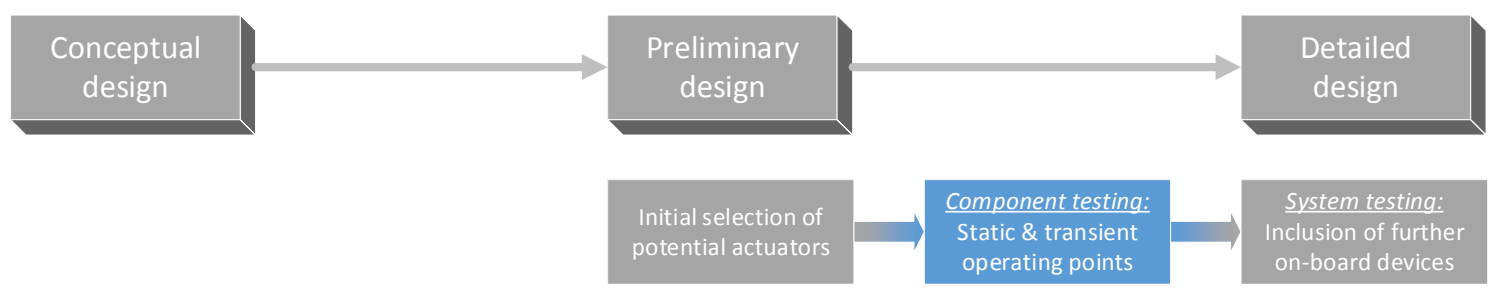

Fig. 1 Actuator testing - integration into the aircraft design process

The test bench is configurable to three operating modes. In Fig. 2 the main arrangement (Mode A) is shown. UAV components are marked in blue and test equipment is marked in orange color. The actuator-under-test is connected to the control surface linkage as in the original aircraft, while the load motor is acting at the hinge axis. The load motor is controlled by Pulse Width Modulation (PWM). The Pulse Pause Modulation (PPM) signal denotes the commanded position value to the actuator. A torque sensor measures the applied torque and a current sensor measures the electric current to the UAV actuator. Angular information is obtained by an incremental encoder inside the load motor. Limit switches assure that control surface travel does not exceed preset boundaries.

By contrast, in Mode B the actuator is directly coupled to the load motor. This topology enables tests focused on the actuator performance without the mechanical linkage influence. Here, actuator angular position is equal to the load motor angular position (assuming a rigid intermediate coupling). In Mode $\mathrm{C}$ the actuator is solely connected to a low-inertia angular position sensor. By providing a quasi-force-free environment, this arrangement can be useful for modelling purposes. A schematic of these configurations is illustrated in section VI. 

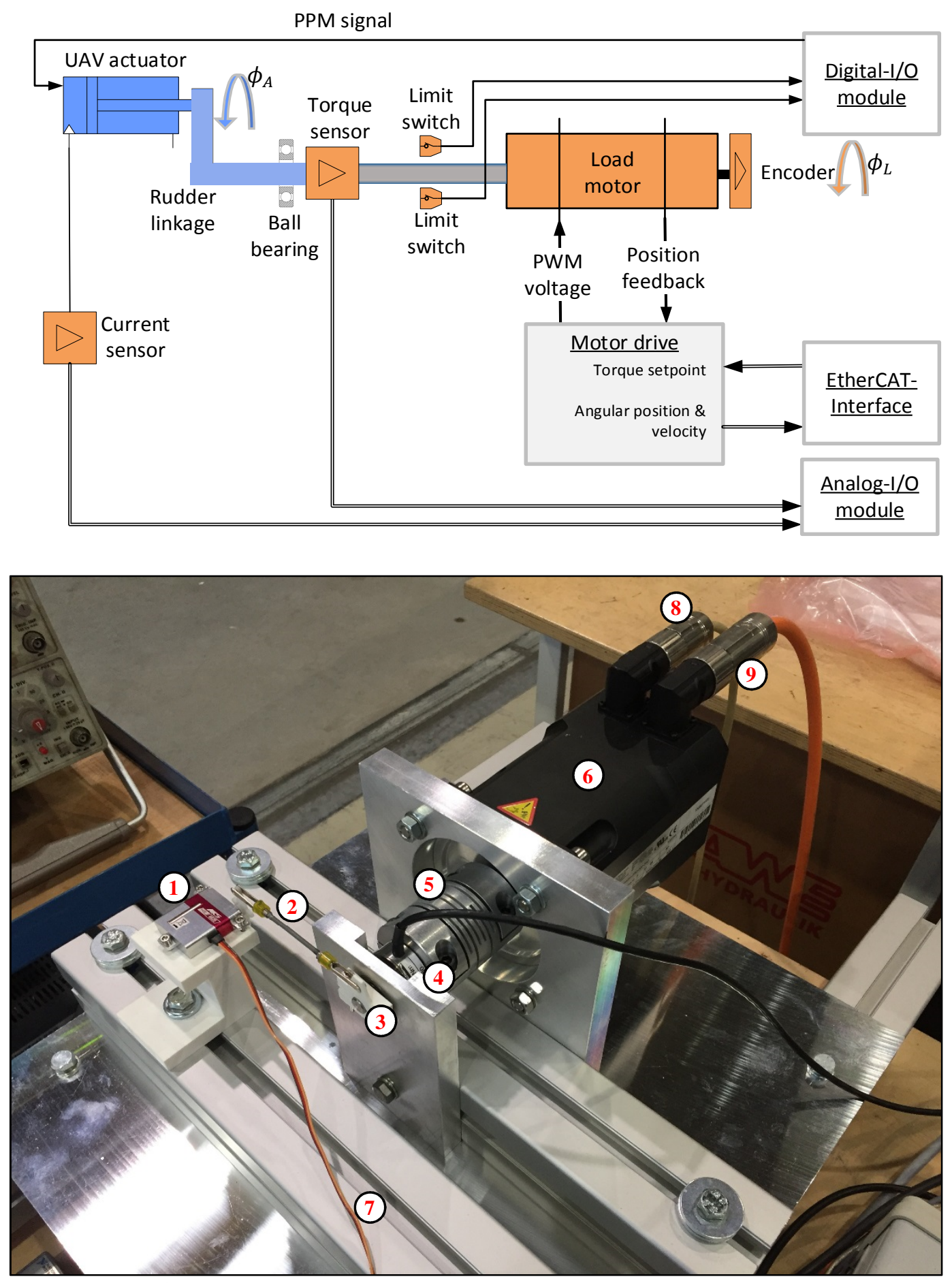
1: UAV actuator-under-test
2: Empennage rudder linkage
3: Ball-bearing mount
4: Torque sensor
5: Beam coupling
6: Load emulation motor
7: UAV actuator power supply \& command signal
8: Load motor position encoder signal
9: Load motor power supply (pulse-width modulated)

Fig. 2 Schematic overview of the experimental setup (Mode A) 


\section{Design considerations}

\section{A. Load emulation}

Selection of an adequate load motor and the accompanying motor drive is based on numeric calculations. First, the expected aerodynamic hinge moment is estimated with XFLR5 along a grid of aircraft speeds and rudder deflection angles ${ }^{5}$. These simulations are based on the planform of a representative UAV currently in development at the institute. Afterwards, the results are packaged in a look-up table and placed into a SIMULINK model, together with simulation blocks of the motion system elements (rectifier, pulse width modulation, and commutation). In this way, the test bench can be simulated at different conditions, with various candidate motors ${ }^{6}$. Usage of a reduction gear for lowering the necessary power rating of the motor is intentionally avoided, by concerns over gear backlash causing torque interruption and the increased reflected inertia "felt" by the UAV actuator.

Besides the performance requirements, emphasis is given to the communication options to/from the motor drive and its control loop update rate. In order to ensure robust signal transmission, a brushless motion system supporting Ethernet for Control Automation Technology (EtherCAT) network capability is chosen.

\section{B. Structural elements}

Theoretically, the applied torque is proportional to the load motor current. However, this hypothetical value is altered along the transmission path to the actuator by friction, mass inertia, temperature variation, and cogging effects. For that reason, a torque sensor is installed, enabling monitoring and closed-loop control. The selection of this sensor is made by a trade-off of measurement range, accuracy, form factor, moment of inertia, and torsional stiffness. Similar properties are considered when selecting an intermediate beam coupling dedicated to reduce shear forces due to shaft misalignment. In Mode $\mathrm{A}$, the position measured by the load motor encoder $\phi_{L}$ will differ from the UAV actuator position $\phi_{A}$, because of the rudder linkage. Due to the torsional deformation of the aluminum elements, a position uncertainty is also present in Mode B. Equation (1) illustrates this relation.

$$
\Delta \phi=-\frac{\mathrm{M}}{k_{\text {coupl. }}+k_{\text {sens. }}}
$$

where $k_{\text {coupl. }}$ and $k_{\text {sens. }}$ are the constants of torsional stiffness for the beam coupling and the torque sensor respectively. At an applied moment $\mathrm{M}$ the angular position between actuator and load motor will differ by $\Delta \phi$.

Furthermore, the frequency bandwidth of the setup has been calculated, in order to ensure that dynamic effects, as encountered in aeroelastic phenomena can be reproduced and captured: by treating the elements of the setup as bodies with a mass inertia, serially connected by torsional springs, the system's mechanical impedance is determined. This allows the estimation of the frequency at which the amplitude and phase registered by the torque sensor start to deviate from that acting on the actuator ${ }^{7}$.

\section{Real-time control and monitoring}

A multitude of signals have to be acquired at the test bench: analog torque and current information, digital encoder pulse trains and limit switch states. Furthermore, various signals have to be generated: actuator command signal and enabling/disabling signals for the motor drive. Since the torque control program (and later on a SIMULINK flight dynamics model) must be executed in real-time, a deterministic PCI eXtensions for Instrumentation (PXI) platform is preferred over execution in Windows. Combined with a remote multichannel acquisition/generation chassis, this platform can be expanded to include additional measurement equipment. Ethernet communication is used for data transfer to the host computer, where user interfacing and parameter monitoring takes place. 


\section{Aerodynamic calculations}

Estimates of the aerodynamic hinge moment expected during flight are used for selecting a load motor that matches the actuator under consideration. During tests, they can be further used for applying a position-dependent load in real-time. The aircraft and mission described in the work of Stahl ${ }^{8}$ serve as a reference for the necessary aerodynamic calculations. This section describes the procedure for the aircraft's empennage actuator.

\section{A. Reference aircraft and flight mission}

The reference UAV demonstrator serves the goal of investigating aeroelastic properties of highly flexible wings. A total of eight aileron and two flutter actuators are installed at the wings. The aircraft features a V-shaped empennage equipped with four ruddervators, each driven by one actuator. Two additional servo actuators drive the air brake flaps.

The highest dynamic pressure $q$ is encountered during steady flight at an airspeed of $V=64 \mathrm{~m} / \mathrm{s}$ and an air density of $\rho=1.225 \mathrm{~kg} / \mathrm{m}^{3}$. It is assumed that maximum stationary loads at the empennage actuators are achieved at this flight state, at full downward deflection $\left(\eta=10^{\circ}\right)$ and maximum angle of attack $\left(\alpha=15^{\circ}\right)$. Longitudinal trim is therefore neglected, in order to achieve a conservative estimate. Transient loads depend on the control surface deflection rate and mass distribution i.a. and are not explicitly calculated in this study.

\section{B. Hinge moment modelling}

The aerodynamic hinge moment results from the difference in pressure distribution between the lower and upper control surface sides. It can be mapped against the angle of attack and flap deflection angle. Possible dynamic pressure increase due to the propulsion system, flow interference between the adjacent control surfaces, and the effect of the finite span might alter the actual hinge moment, but are not taken into account in the scope of this work. The magnitude of the hinge moment $M$ is defined in Eq. (2).

$$
M=\frac{1}{2} \rho V^{2} \cdot \int_{y=0}^{b_{f}} \int_{x=0}^{x_{T . E .}} x \cdot\left(C_{p, u}-C_{p, l}\right) d x d y
$$

where $\rho$ is the air density and $V$ is the airspeed. $C_{p, u}$ and $C_{p, l}$ stands for the pressure coefficient distribution along the upper and lower control surface side respectively. The direction of the hinge moment is visible in Fig. 3.

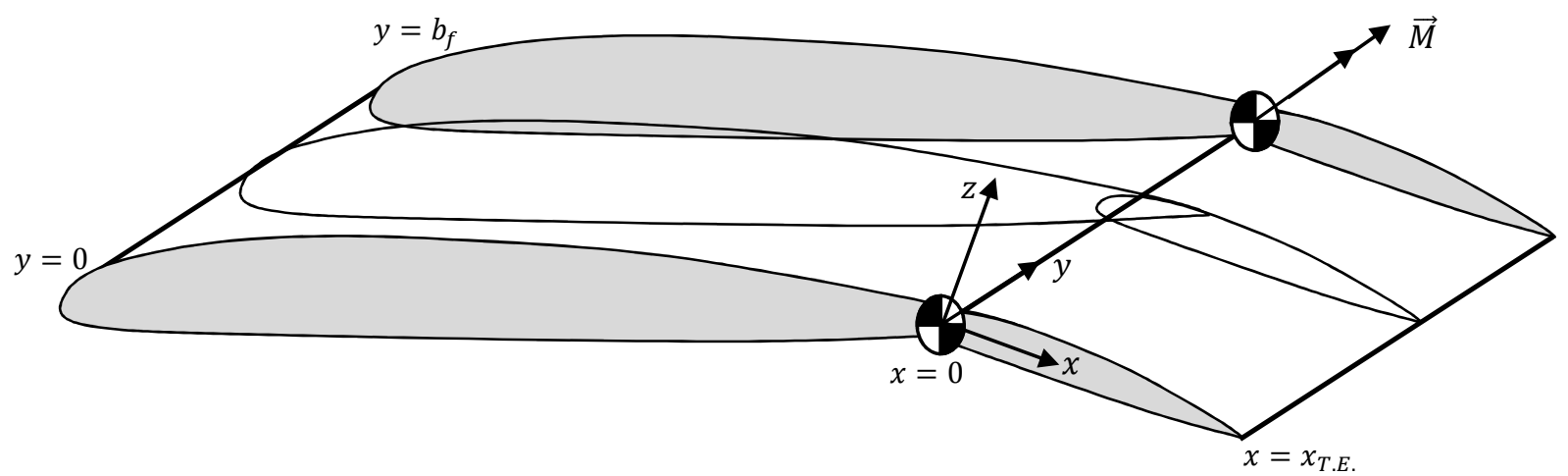

Fig. 3 Detail of the empennage control surface

XFLR5 returns the hinge moment coefficient per span $C_{H M}$. Thus, the hinge moment is obtained by Eq. (3).

$$
M=-\frac{1}{2} \rho V^{2} \cdot C_{H M} \cdot c_{e m p}^{2} \cdot b_{f}
$$

Numeric calculations are performed at the Reynolds and Mach numbers corresponding to the airspeeds $V=32 \mathrm{~m} / \mathrm{s}$ and $V=64 \mathrm{~m} / \mathrm{s}$. The results of the calculations are displayed in Fig. 4. At high angles of attack and/or control surface deflection, the simulation does not converge (highlighted in red), possibly due to flow separation. Linear extrapolation from converged points is used to estimate the missing values. 

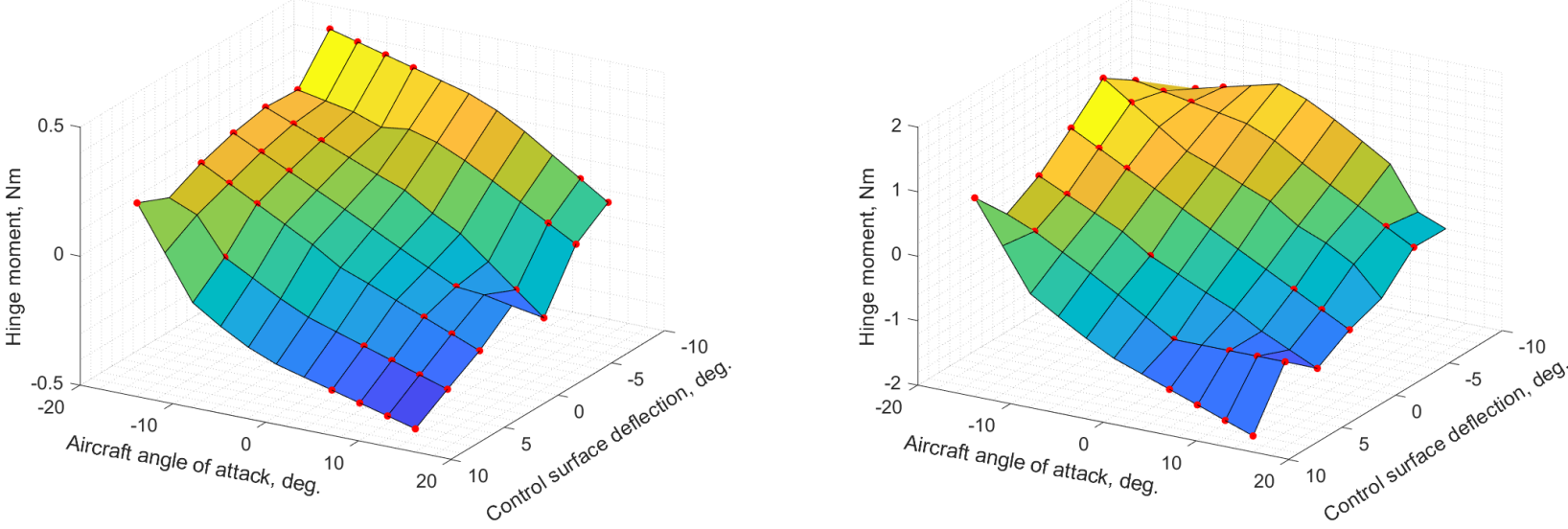

left: $V=32 \mathrm{~m} / \mathrm{s}\left(\operatorname{Re}=6.5 \cdot 10^{5}, M a=0.09\right)$

right: $V=64 \mathrm{~m} / \mathrm{s}\left(\operatorname{Re}=1.3 \cdot 10^{6}, M a=0.19\right)$

Fig. 4 Hinge moment dependence on control surface deflection and angle of attack

These calculations are performed in order to choose an appropriate load motor for the empennage actuator. The load motor for the air brake actuator was chosen based on an already existing study?

\section{Instrumentation}

This section gives a technical overview of the sensors and actuators installed in the experimental setup, as well as the instrumentation hardware and software used.

\section{A. Sensors}

Following physical quantities are acquired and recorded for post-processing:

Torque: The mechanical load acting on the actuator is measured by resistance strain gauges. The sensor consists of an inner and outer ring connected to the actuator and load motor respectively. Four spokes equipped with strain gauges connect the two rings radially, acting as bending beams. Three torque classes are available: a small and medium one for low torque levels (primary control system actuators) and a larger one for higher torque levels (secondary control system actuators). All feature Wheatstone bridge circuits in full configuration.

Current: A magneto-resistive element measures the instantaneous electric current flow to the aircraft actuator by the magnetic field generated inside the sensor housing. The sensor is placed in series with the low-side (ground terminal) of the actuator power supply unit.

Voltage: The applied electric voltage at the actuator terminals is recorded for power supply calculations and for locating possible voltage drops across the actuator power supply cabling.

Angular movement: In Mode A \& B an incremental EnDat optical encoder, integrated in the load motor casing computes the angular position and velocity of the rotor. The load motor also features built-in winding current and temperature monitoring. For tests without the load motor (Mode C), an external optical encoder is used instead. An overview of the sensors of the test bench can be found in Table 1 .

Table 1. Test bench sensors

\begin{tabular}{|c|c|c|c|c|}
\hline & $\begin{array}{c}\text { Measurement } \\
\text { principle }\end{array}$ & Measurement range & $\begin{array}{c}\text { Sensor accuracy } \\
\text { (specifications) }\end{array}$ & Acquisition module \\
\hline Torque & $\begin{array}{l}\text { Resistance strain } \\
\text { gauge }\end{array}$ & $\begin{array}{c}{[-5,+5] \mathrm{Nm}} \\
{[-10,+10] \mathrm{Nm}} \\
{[-20,+20] \mathrm{Nm}}\end{array}$ & $\begin{array}{c}0.005 \mathrm{Nm} \\
0.01 \mathrm{Nm} \\
0.02 \mathrm{Nm} \\
\end{array}$ & analog input (24-bit, $\delta-\sigma)$ \\
\hline $\begin{array}{l}\text { Electric } \\
\text { current }\end{array}$ & $\begin{array}{l}\text { Mangeto-resistive } \\
\text { effect }\end{array}$ & {$[-15,+15] \mathrm{A}$} & $0.12 \mathrm{~A}$ & analog input (16-bit, SAR ) \\
\hline Voltage & Comparator bank & {$[-10,+10] \mathrm{V}$} & $0.06 \mathrm{~V}$ & analog input (16-bit, $\delta-\sigma)$ \\
\hline $\begin{array}{l}\text { Angular } \\
\text { position }\end{array}$ & \multirow{2}{*}{$\begin{array}{l}\text { Optical encoder } \\
\text { (for Mode A \& B) }\end{array}$} & {$[-180,+180]$ deg. } & $5.6 \cdot 10^{-3} \mathrm{deg}$. & \multirow{2}{*}{ Load motor drive } \\
\hline $\begin{array}{l}\text { Angular } \\
\text { velocity }\end{array}$ & & {$[-3000,+3000]$ deg./s } & $8.8 \cdot 10^{-8} \mathrm{deg} . / \mathrm{s}$ & \\
\hline $\begin{array}{l}\text { Angular } \\
\text { position }\end{array}$ & $\begin{array}{l}\text { Optical encoder } \\
\text { (for Mode C) }\end{array}$ & {$[-180,+180]$ deg. } & 0.35 deg. & Digital input (TTL) \\
\hline
\end{tabular}




\section{B. Actuators}

Two actuator types are used in this test bench: a rotary servo actuator of the UAV under consideration and an aerodynamic load emulation actuator, simply referred to as load motor. Both are brushless DC electric motors (Table 2). The former is supplied by direct current and the latter by rectified three-phase alternating current.

The UAV actuator is equipped with a gearbox and a potentiometer-based position sensor. It is commanded by the PPM technique, exemplified in Fig. 5. A repetitive pulse with a "high" duration ranging between $0.9 \mathrm{~ms}$ and $2.1 \mathrm{~ms}$ is interpreted by the actuator electronics as the desired angular position. The longest one corresponds to the shaft position of $\phi_{A}=0^{\circ}$ (flap fully retracted) while the shortest corresponds to the shaft position of $\phi_{A}=117^{\circ}$ (fully extended flap). Intermediate positions are reached by adjusting the duration linearly. A digital output module generates the described signal with a period of $3.0 \mathrm{~ms}$ instead of the radio controller or FCC. In this way, manual or automatic (step/sine patterns) setpoint adjustment is possible.

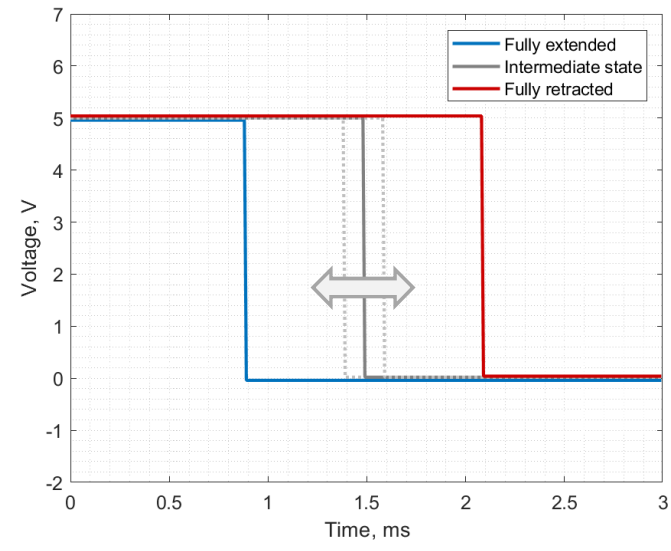

Fig. 5 UAV actuator command signal

Table 2. Test bench actuators

\begin{tabular}{|c|c|c|c|c|c|}
\hline & Operating principle & Position feedback & $\begin{array}{c}\text { Continuous } \\
\text { stall } \\
\text { torque }[\mathrm{Nm}]\end{array}$ & $\begin{array}{c}\text { Instantaneous } \\
\text { peak } \\
\text { torque }[\mathrm{Nm}]\end{array}$ & $\begin{array}{c}\text { Rotor moment } \\
\text { of inertia } \\
{\left[\mathrm{kg} \cdot \mathrm{m}^{2}\right]}\end{array}$ \\
\hline $\begin{array}{l}\text { UAV air brake } \\
\text { actuator }\end{array}$ & $\begin{array}{l}\text { Permanent magnet } \\
\text { DC motor (brushless) }\end{array}$ & $\begin{array}{l}\text { Integrated resistance } \\
\text { potentiometer }\end{array}$ & 5.9 & 19.6 & n.a. \\
\hline $\begin{array}{l}\text { Load emulation } \\
\text { motor }\end{array}$ & $\begin{array}{l}\text { Permanent magnet } \\
\text { DC motor (brushless) }\end{array}$ & $\begin{array}{l}\text { Integrated optical } \\
\text { encoder }\end{array}$ & 8.4 & 21.3 & $6.2 \cdot 10^{-4}$ \\
\hline
\end{tabular}

\section{Measurement hardware and software}

The actuator test bench is connected to a local measurement chassis housing the hardware modules. These acquire the torque, current, voltage, as well as generate the PPM control signal and physical drive-enabling signals. This chassis is a slave node of a broader EtherCAT ring network, which also includes the load motor drive. Program execution, data processing, and parameter recording takes place in the PXI computer which is the master node of the network, as denoted in Fig. 6. Communication is established by a datagram passing the participating nodes circularly. Each one receives the corresponding data and/or places timestamped data onto the telegram. This architecture is arbitrary scalable and enables short analog conductor paths instead of lengthy ones, which are prone to signal degradation and electromagnetic interference (EMI) complications. Furthermore, the motor drive is compatible with the network protocol and can therefore be digitally controlled.

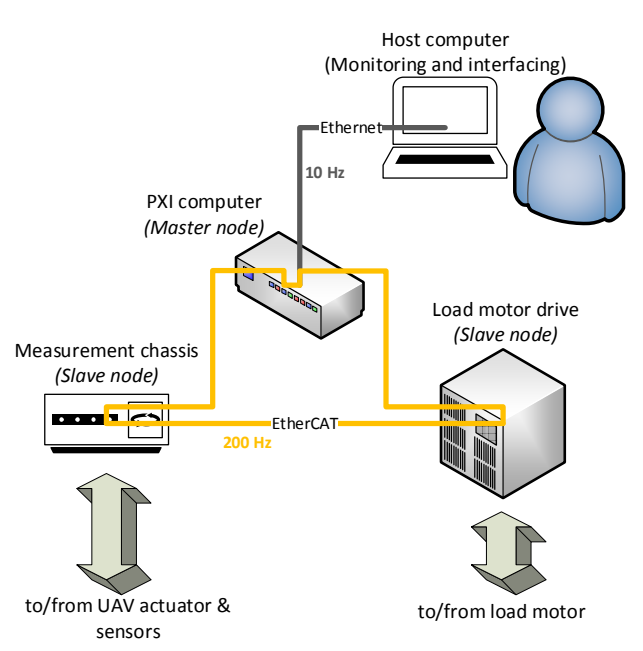

Fig. 6 Communication network

All individual sensor values mentioned above are cyclically acquired with a scan frequency of $f_{\text {scan }}=200 \mathrm{~Hz}$ (higher scan rates up to $1000 \mathrm{~Hz}$ are proven to be feasible). Signals demanding faster signal processing, e.g. PPM and encoder position are handled by a Field Programmable Gate Array (FPGA) with an update frequency of $f_{P P M}=333 \mathrm{~Hz}$ and $f_{\text {enc. }}=40 \mathrm{kHz}$ respectively. The PXI platform is connected to a conventional personal computer, where user interfacing is performed in a LABVIEW environment. There, monitor and command values are exchanged periodically at a slower rate. A state machine program handles the transition between the individual test states: initialize, idle, test run, stop, and data storage. The transition conditions are based on user input and logical signal values, e.g. ready-to-operate signal from the load motor and limit switch information. 


\section{Test conduction and results}

\section{A. Experimental procedure}

In this section, the test procedure for the air brake servo actuator is presented. The FLEXOP demonstrator aircraft is equipped with an air brake system for aiding airspeed control. Each actuator extends/retracts a fuselagemounted flap by moving an intermediate linkage. Two scenarios had to be addressed: behavior of the actuator when commanded to hold neutral position and when commanded to alternate between two specified positions in a step-wise fashion. The torque is to be held constant, but is varied from test to test, as does the duration between the position command switch. Table 3 summarizes the test campaign in tabular form. The experimental setup is visible in Fig. 7. While the static test conditions are based on the same study which was used for the selection of the load motor, the transient test conditions are based on the demands of the autopilot design team.

Table 3. Test matrix for UAV air brake actuator

\begin{tabular}{|c|c|c|c|c|c|c|}
\hline & & Static tests & \multicolumn{4}{|c|}{ Transient tests } \\
\hline \multirow{2}{*}{$\begin{array}{l}\text { UAV actuator } \\
\text { settings }\end{array}$} & Position setpoint [ $\mathrm{deg}]$. & 58.5 & $0 \leftrightarrow 29.25$ & $0 \leftrightarrow 58.5$ & $29.25 \leftrightarrow 58.5$ & $0 \leftrightarrow 117$ \\
\hline & Switching frequency $[\mathrm{Hz}]$ & $\{0\}$ & \multicolumn{4}{|c|}{$2,4,6,8,10$} \\
\hline $\begin{array}{l}\text { Load motor } \\
\text { settings }\end{array}$ & Torque setpoint $[\mathrm{Nm}]$ & $\begin{array}{c}\{1,2,3,4,5,6,7,8\} \\
\text {-Mode B- }\end{array}$ & & $\begin{array}{l}\{0\} \quad \& \\
\text {-Mode C- }\end{array}$ & $\begin{array}{l}\{2,4,6,8\} \\
\text {-Mode B- }\end{array}$ & \\
\hline
\end{tabular}
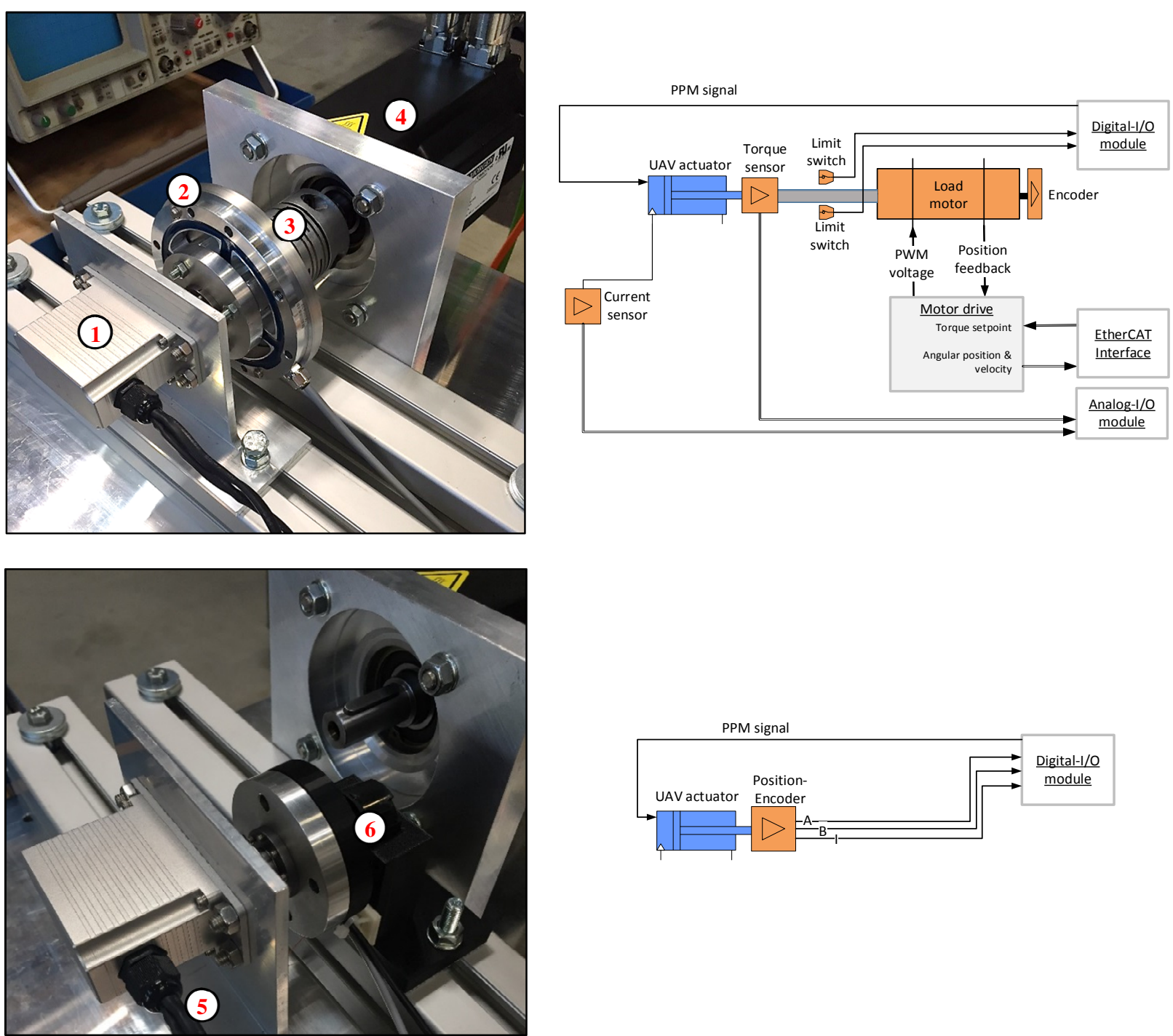

1: UAV actuator-under-test, 2: Torque sensor, 3: Beam coupling, 4: Load motor, 5: UAV actuator power supply \& command, 6: optical encoder

Fig. 7 Schematic overview of the experimental setup (Mode B and Mode C) 


\section{B. Calibration and tuning}

Temperature variation, mechanical hysteresis, and degradation of electronic components will lead to inaccurate torque and current readings. Hence, the offset and slope errors of the torque and current sensors are periodically corrected. A weight of known mass and an adjustable current source respectively are utilized for the two-point calibration (Fig. 8). In order to counter measurement noise during calibration a moving average is implemented. Later, during actual tests, the raw data torque value is recorded instead. Next, the relationship between the load motor setting and the torque reading is established by blocking the torque sensor and successively raising the motor output. The resulting look-up table enables the direct application of a desired torque level.
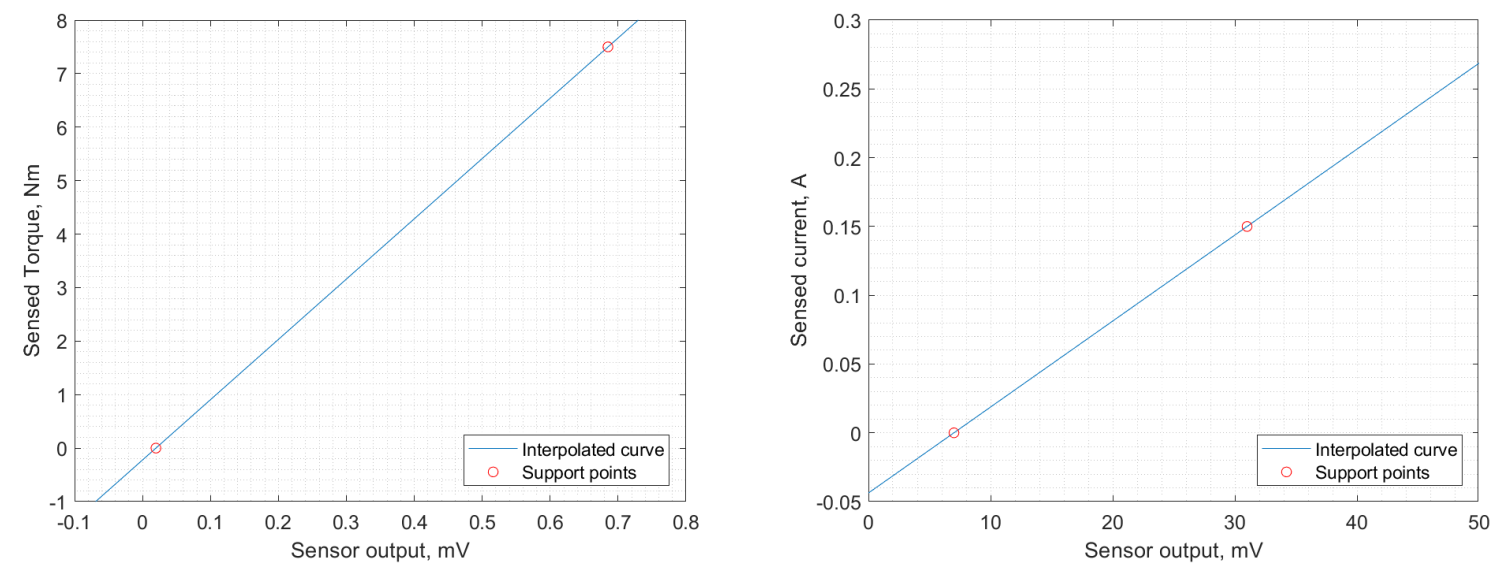

Fig. 8 Calibration curves of torque and current sensor

An un-energized motor will display a certain mechanical resistance when rotated by an external force, an effect known as cogging. The load motor drive software is equipped with a compensation option for active reduction of the unwanted cogging torque. After a teach-in sequence with a free moving shaft at low speed, a compensation table is automatically generated. Depending on the instantaneous rotor position and movement direction, a specific amount of additional current is superimposed during actual tests in order to reduce cogging. This aims to improve the application of the desired torque and allow for low torque levels. Nonetheless, tests reveal that complete suppression of the effect is not achieved.

\section{Results}

Load application is performed by adjusting the current setting to the load motor. In combination with a feedforward path based on the static current/torque relationship, a feedback path taking into account the actual torque reading is implemented.

Static performance: while the actuator is commanded to hold its neutral position $\left(\phi_{A}=58.5^{\circ}\right.$, refer to section V.B), the load level is increased stepwise by $1 \mathrm{Nm}$ every 3 seconds. On the left chart of Fig. 9 the measured torque is shown. On the middle chart the angular displacement of the actuator during loading is displayed. The rightmost chart shows the actuator current draw. Following the convention of section II, graphs indicating UAV actuator performance are marked with a blue triangle, while curves representing the test bench characteristics are marked with an orange triangle. The grey dots connect the mean values of the discrete measurement points, while the blue bars indicate the standard deviation at these measurement points over time for torque and position. Skewness in the current data renders the standard deviation measure ineffective here, therefore an average deviation from the mean is calculated for values higher and lower than the mean current separately.

- It is visible that the measured torque value differs only slightly from the commanded one. The standard deviation however increases at higher load levels.

- The position controller of the UAV actuator does not fully compensate for external loads (the theoretical sensor and coupling deformation is also calculated and proven to be negligible in this range). Nevertheless the position signal is steady even at high loads, as can be seen by the standard deviation.

- Although the actuator power supply unit indicates a steady current during loading, the measured current shows high peaks in reality, possibly due to the actuator circuit blocking and releasing the input current periodically.

At a torque of $M=8.5 \mathrm{Nm}$ overheat of the load motor occurred. Slightly under this value, the actuator shaft started moving, since it cannot hold the commanded position. 

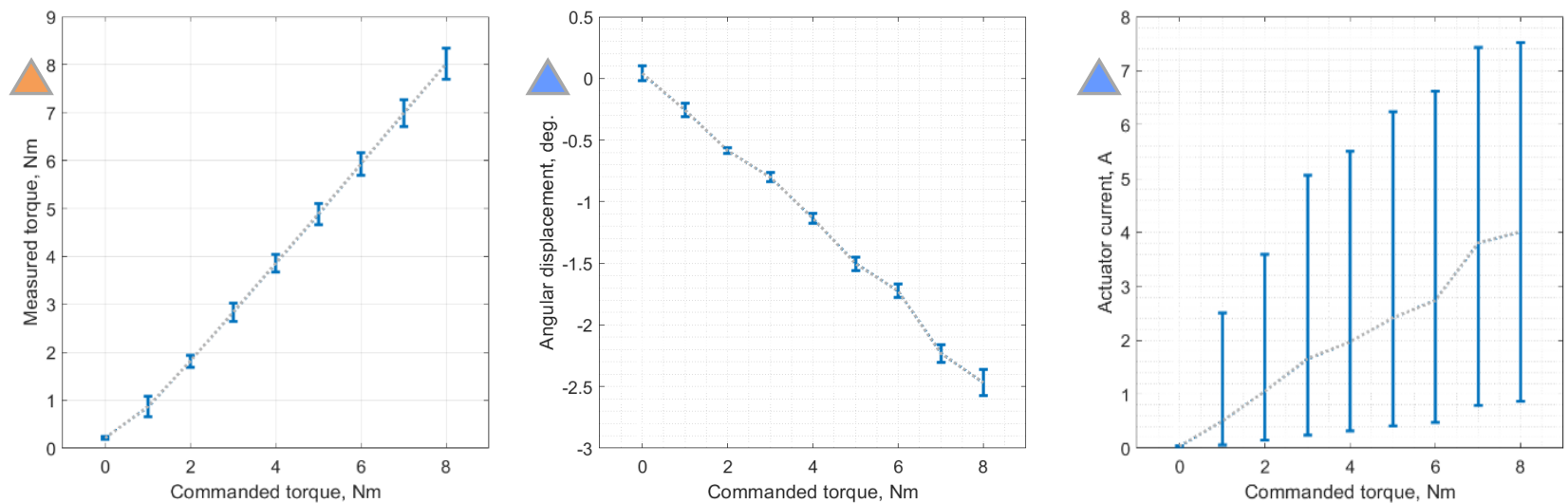

Fig. 9 Static actuator response to different load levels

Transient performance: Excerpts from the transient tests are illustrated in Fig. 10 and Fig. 11. In the left diagrams, the resistive torque is shown. The middle and right diagrams show the angular movement of the shaft, in position and velocity respectively. In the loading phase, the velocity is significantly reduced, since resistance and movement oppose each other. The velocity is increased when the two quantities have the same sign, as expected. However, a fluctuation in torque is visible throughout the test. It is probably attributed to cogging in the load motor and the torque control loop not completely cancelling torque peaks due to load motor inertia.
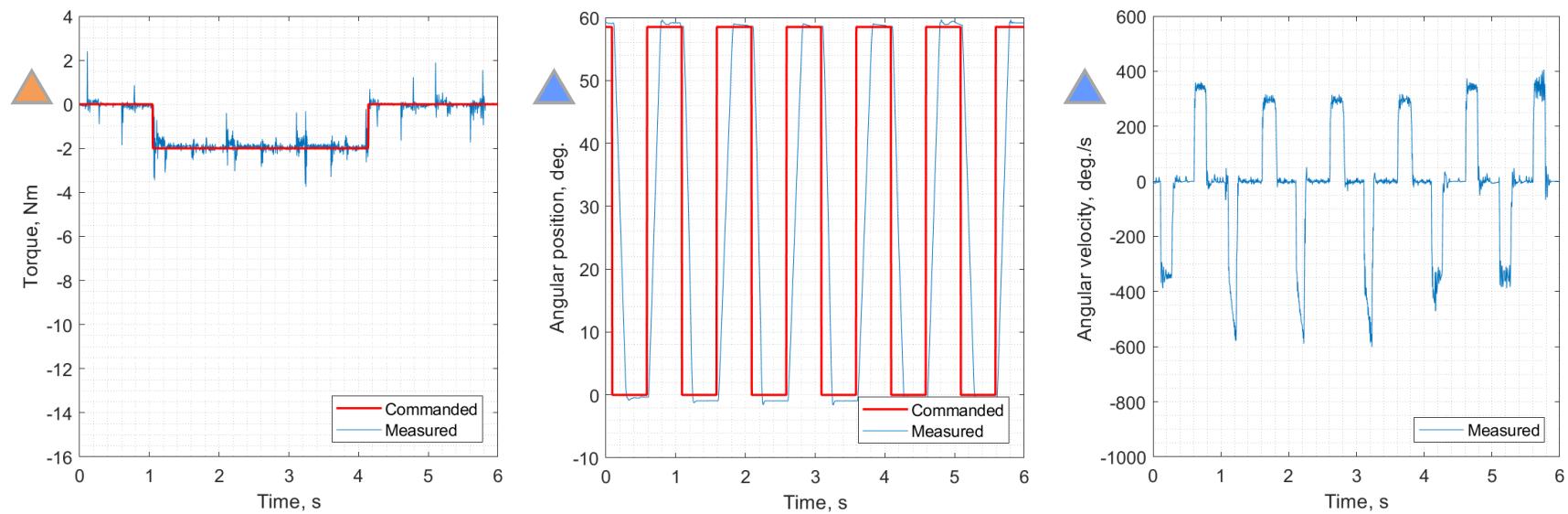

torque setpoint: $2 \mathrm{Nm}$, switching frequency: $2 \mathrm{~Hz}$, position setpoint range: $0^{\circ} \leftrightarrow 58.5^{\circ}$

Fig. 10 Mode B measurement (example 1)
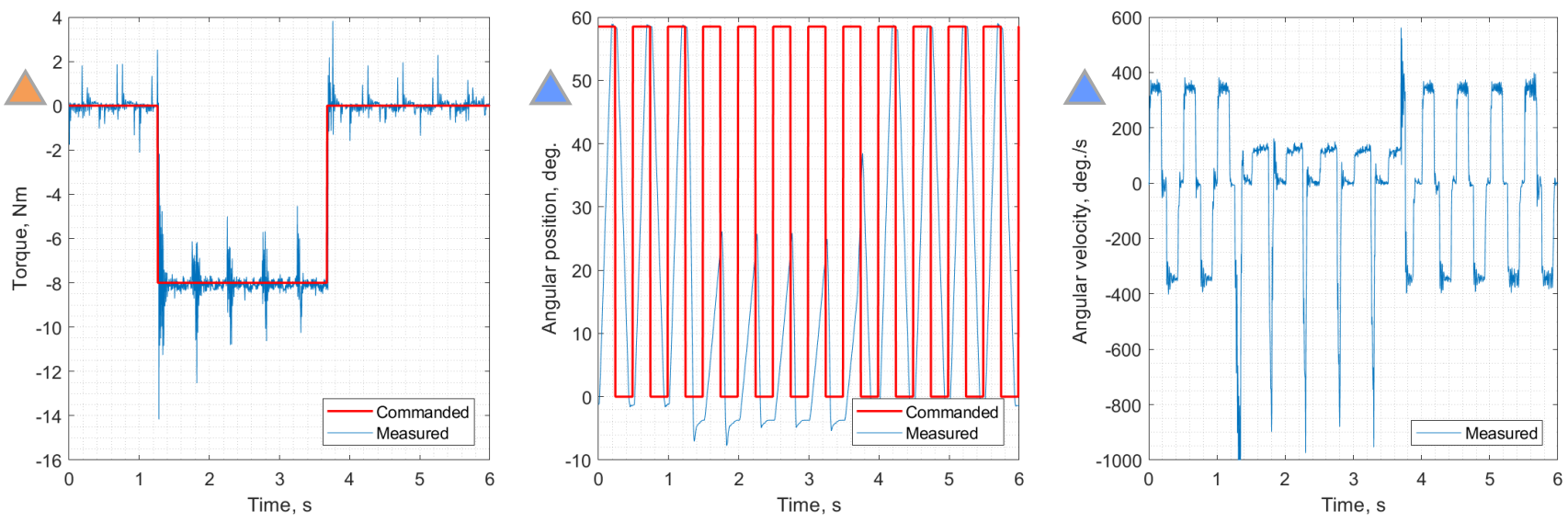

torque setpoint: $8 \mathrm{Nm}$, switching frequency: $4 \mathrm{~Hz}$, position setpoint range: $0^{\circ} \leftrightarrow 58.5^{\circ}$

Fig. 11 Mode B measurement (example 2) 


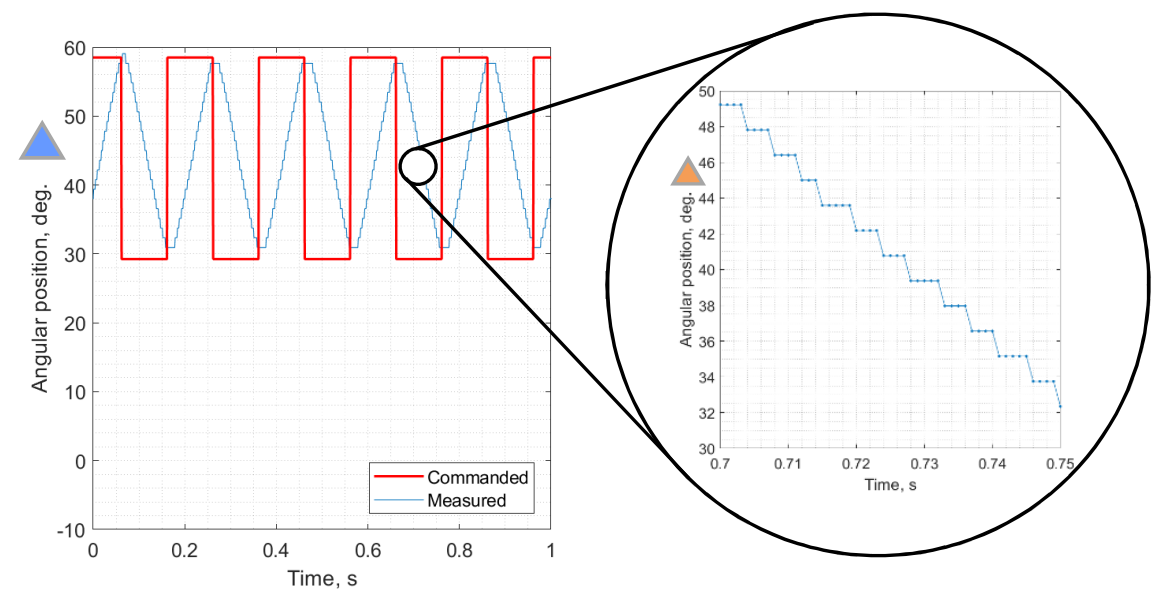

switching frequency: $10 \mathrm{~Hz}$, position setpoint range: $29.25^{\circ} \leftrightarrow 58.5^{\circ}$

Fig. 12 Mode $\mathrm{C}$ measurement

In Fig. 12, an excerpt from the trials without the load motor is presented. They were carried out in order to support the previous measurements at zero torque, since the load motor is absent in this arrangement. However, extracting velocity information proves to be challenging, due to the external position encoder output remaining constant until a new line is registered by the optical element.

Fig. 13 attempts to convey a picture of the actuator capabilities at different applied load levels and different position switching frequencies. The achievable angular velocity (z-axis) decreases with increasing applied load, but is only marginally affected by the position switching frequency: this is due to the fact that the UAV actuator achieves a steady velocity in a much shorter time than the setpoint variation. However, the angular position range will decrease with increasing switching frequency, as the setpoint changes before it is achieved by the actuator. In real flight, the load will generally increase with rudder deflection, however the load was commanded to stay constant during the tests. Nevertheless, extracting further actuator characteristics, e.g. conservative estimates of the required duration for achieving a specific flap deflection during flight maneuvers, is feasible.
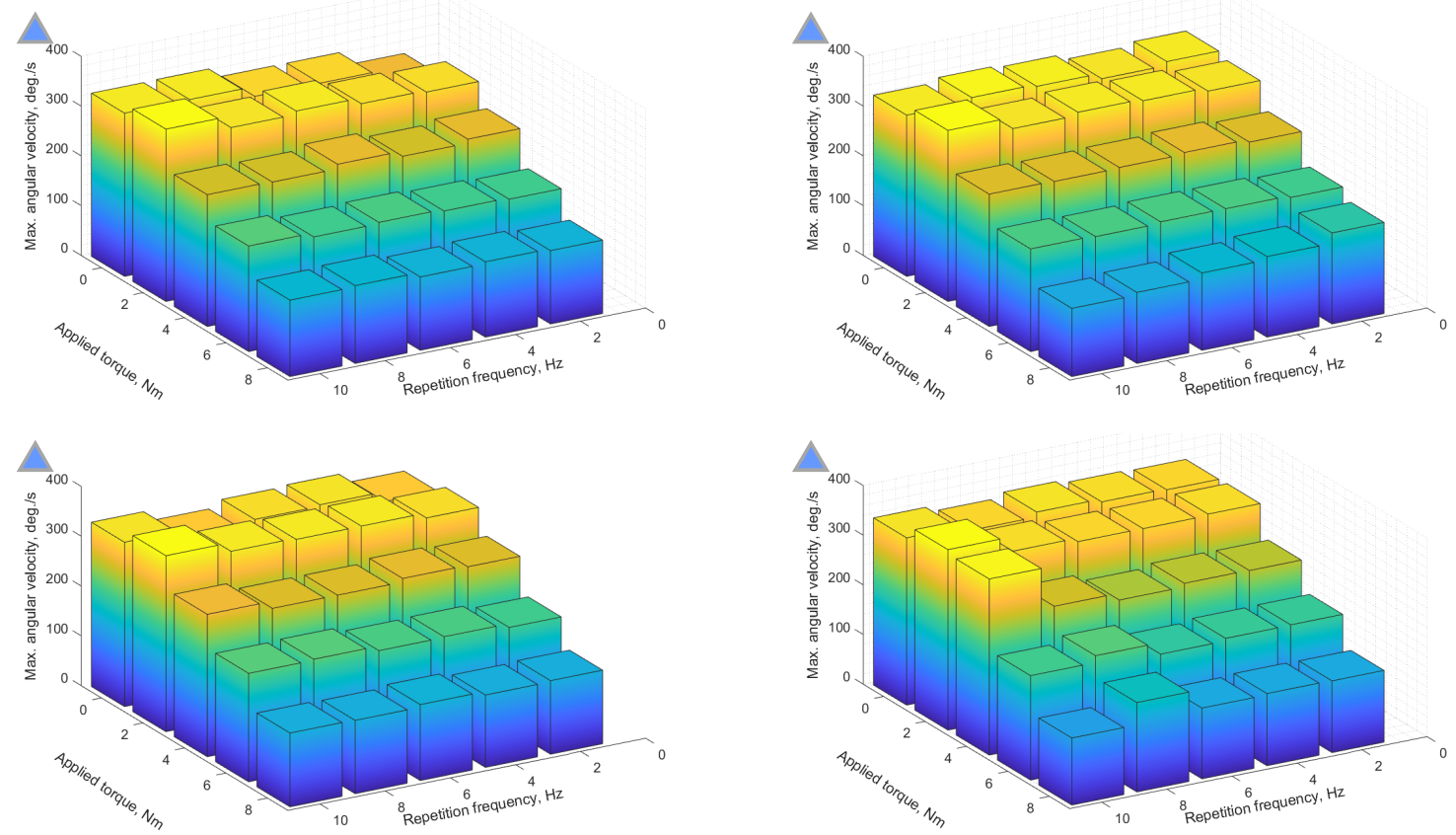

position setpoint range:

left upper: $0^{\circ} \leftrightarrow 29.25^{\circ}, \quad$ right upper: $0^{\circ} \leftrightarrow 58.5^{\circ}$

left lower: $29.25^{\circ} \leftrightarrow 58.5^{\circ}$ right lower: $0^{\circ} \leftrightarrow 117^{\circ}$

Fig. 13 Synopsis of actuator transient response 


\section{Conclusion}

The presented test bench offers a modular platform for testing electro-mechanical UAV servo actuators by aerodynamic load emulation and load measurement. Performance characteristics, such as position-following error and velocity are quantified. Furthermore, continuous and peak electric current demand by the actuator is calculated. Special technical features of the experimental setup are: distributed signal acquisition/generation for shortened analog transmission paths, synchronized communication network for common-timestamp data recording, and active cogging reduction for torque handling.

Results of this study could be utilized by an external FLEXOP project partner when designing the autopilot module for the air brake.

Although the intended function of the test bench is proven, certain issues remain, primarily handling unwanted torque fluctuations. Additionally, the aerodynamic hinge moment calculations often fail to converge at operating points that have an impact on load motor selection. Future tasks will be:

- improvement of torque control

- implementation of position-dependent load application in real-time

- improvement of aerodynamic calculations

- examination of the electric current and voltage signal at transient tests

\section{Acknowledgment}

The research leading to these results is part of the FLEXOP project. This project has received funding from the European Unions Horizon 2020 research and innovation program under grant agreement No 636307. The work of this paper will actively contribute to the FLEXOP project but has been funded by TUM internal resources.

\section{References}

${ }^{1}$ Mare, J.-C., "Dynamic loading systems for ground testing of high speed aerospace actuators," Aircraft Engineering and Aerospace Technology, Vol. 78, No. 4, 2006, pp. 275-282.

${ }^{2}$ Lauffs, P. J., and Holzapfel, F., "Hardware-in-the-loop platform for development of redundant smart actuators," Aircraft Engineering and Aerospace Technology, Vol. 88, No. 3, 2016, pp. 358-364.

${ }^{3}$ Braun, R., "Hardware-in-the-loop simulation of aircraft actuator," Master thesis, Institute of Technology, Linköping, Sweden, 2009.

${ }^{4}$ Bertucci, A., Mornacchi, A., Jacazio, G., and Sorli, M., “A Force Control Test Rig for the Dynamic Characterization of Helicopter Primary Flight Control Systems," Procedia Engineering, Vol. 106, 2015, pp. 7182.

5Zimmermann, C., "CAD construction and FEM analysis of a UAV test bench," Bachelor thesis, Institute of Aircraft Design, Technical University of Munich, Garching, Germany, 2017.

${ }^{6}$ Yang, J., "Research study on electrical motors for an UAV-test bench and simulation of operation," Bachelor thesis, Institute of Aircraft Design, Technical University of Munich, Garching, Germany, 2017.

${ }^{7}$ Jiahe, L., "Research study on torque sensors for a UAV-test bench," Bachelor thesis, Institute of Aircraft Design, Technical University of Munich, Garching, Germany, 2017.

${ }^{8}$ Stahl, P., Sendner, F. M., Hermanutz, A., Rößler, C., and Hornung, M., "Mission and Aircraft Design of FLEXOP Unmanned Flying Demonstrator to Test Flutter Suppression within Visual Line of Sight," 17th AIAA Aviation Technology, Integration, and Operations Conference, American Institute of Aeronautics and Astronautics, Reston, Virginia, 2017.

${ }^{9}$ Koerner, D., "Experimental System Identification of an Electric Actuated Airbrake System for the FLEXOP Research UAV," Bachelor thesis, Institute of Aircraft Design, Technical University of Munich, Garching, Germany, 2018. 